\title{
A low-complexity PAPR reduction SLM scheme for STBC MIMO-OFDM systems based on constellation extension
}

\author{
Guang $\mathbf{L i}^{{ }^{*}}$, Tianyun $\mathbf{L i}^{1}$ \\ ${ }^{1}$ National Digital System Engineering and Technological Research Center \\ ZhengZhou 450000, China \\ [e-mail: 1226813282@qq.com] \\ *Corresponding author: Guang Li
}

Received October 21, 2018; revised December 1, 2018; accepted January 14, 2019; published June 30, 2019

\begin{abstract}
Multiple input multiple output orthogonal frequency division multiplexing (MIMO-OFDM) is widely applied in wireless communication by virtue of its excellent properties in data transmission rate and transmission accuracy. However, as a major drawback of MIMO-OFDM systems, the high peak-to-average power ratio (PAPR) complicates the design of the power amplifier at the receiver end. Some available PAPR reduction methods such as selective mapping (SLM) suffer from high computational complexity. In this paper, a low-complexity SLM method based on active constellation extension (ACE) and joint space-time selective mapping (AST-SLM) for reducing PAPR in Alamouti STBC MIMO-OFDM systems is proposed. In SLM scheme, two IFFT operations are required for obtaining each transmission sequence pair, and the selected phase vector is transmitted as side information(SI). However, in the proposed AST-SLM method, only a few IFFT operations are required for generating all the transmission sequence pairs. The complexity of AST-SLM is at least $86 \%$ less than SLM. In addition, the SI needed in AST-SLM is at least $92.1 \%$ less than SLM by using the presented blind detection scheme to estimate SI. We show, analytically and with simulations, that AST-SLM can achieve significant performance of PAPR reduction and close performance of bit error rate (BER) compared to SLM scheme.
\end{abstract}

Keywords: Active constellation extension, peak-to-average power ratio, cyclic shift, weighted combination 


\section{Introduction}

Multiple input multiple output orthogonal frequency division multiplexing (MIMO-OFDM) is an efficient technique for wireless communication, which utilizes spatial multiplexing technology to improve data transmission rate and ensure transmission accuracy via spatial diversity and beamforming [1]. It plays a vital role in many broadband wireless standards, including the IEEE 802.11ac for wireless local networking [2], IEEE 802.16e for metropolitan area networks [3], and third generation partnership project (3GPP) long term evolution (LTE) for cellular networks [4]. Moreover, it has been widely used in the 4G wireless communications and will be applied to the 5G wireless communications [5][6]. Compared with the traditional MIMO-OFDM system, STBC MIMO-OFDM system introduces spatial diversity gain, which enhances the transmission reliability [7]. However, the MIMO-OFDM system usually suffers from high PAPR, which complicates the design of the power amplifier at the receiver end . Therefore, the design of PAPR reduction scheme has become a hot spot in MIMO-OFDM research.

Classical PAPR reduction techniques include clipping, selective mapping (SLM), partial transmission sequence (PTS), and active constellation extension (ACE) [8]-[11]. Some methods, such as clipping, reduce PAPR by performing nonlinear preprocess to time-free signal. Though simple in principle and reducing PAPR significantly, the clipping technology leads to signal distortion and degradation of bit error rate (BER) performance [12]. ACE improves the BER performance by correcting the constellation after clipping [13]. Methods like SLM and PTS, reduce PAPR by changing the phase distribution of data frame. In SLM, the phase distribution of data frame is changed by multipling the data frame with a random phase vector [9]. While PTS divides a large frame into several subblocks, and multiply them with diffirent phase factors [11]. However, both SLM and PTS need to transmit the phase vector or phase factors as side information (SI), which wastes spectrum resource a lot.

Recently, various improved methods have been proposed to alleviate the PAPR problem of MIMO-OFDM. In [14], a novel phase offset SLM scheme has been proposed to reduce the PAPR without SI in Alamouti MIMO-OFDM systems. However, the complexity of this method is high. The work in [15] proposed the using of combination of PTS and signal clipping techniques to solve the high PAPR, but the exhaustive search in PTS process cost large computational complexity and the nonlinear distortion was introduced to system by clipping. In [16], a simple SLM method based on time-domain sequence cyclic shift and combination of antennas is proposed. However, the time-domain sequences generated by this method is not independent, which leads to a poor PAPR reduction performation compared to SLM.

In this work, a joint space-time selective mapping scheme based on active constellation extension, also called AST-SLM, is proposed. Unlike the traditional selective mapping (SLM) scheme, the transmission candidate sequence pairs are generated via weighted combination between the cyclic-shifted sequences in different antennas, and before this step, the active constellation extension is performed to the IFFT-operated time-domain sequences to reduce PAPR for the first time. While requiring only a small amount of computation, this scheme achieve better performance in reducing PAPR than SLM. Moreover, a blind detection scheme is proposed to estimate SI, so the SI needed in AST-SLM is less than SLM.

The rest of the paper is organized as follows. In Section 2, the main theory of Alamouti STBC MIMO-OFDM system, as well as the definition of PAPR are explained, and the SLM 
scheme is also described in this section. In Section 3, the AST-SLM and the blind detection scheme for estimating SI are described. In Section 4, the analysis of computational complexity is introduced. In Section 5, the simulation results and analysis are described. Finally, conclusions are given in Section 6.

\section{Alamouti STBC MIMO-OFDM and SLM Scheme}

\subsection{Alamouti STBC MIMO-OFDM}

We consider a two-antennas Space Time Block Codes (STBC) MIMO-OFDM system that adopts the Alamouti scheme. The orignal OFDM sequence transmitted in each antenna is denoted by:

$$
X_{i}=\left[X_{i}(0), X_{i}(1), \cdots, X_{i}(N-1)\right]
$$

where $N$ is the number of subcarriers, $i$ is the index of antenna. The frame structure of the STBC MIMO-OFDM system is expressed as

$$
X_{\text {STBC }}=\left[\begin{array}{cc}
X_{1} & X_{2} \\
-X_{2}^{*} & X_{1}^{*}
\end{array}\right]
$$

where $(\cdot)^{*}$ indicates complex conjugate. The frames in the first column of $X_{\text {STBC }}$ are transmitted from antenna 1 during the first and the next symbol period, respectively. And the frames in the second column of $X_{\text {SтBC }}$ are transmitted from antenna 2 during the first and the next symbol period, respectively [17].

The time-domain sequence on the antenna $i$ can be expressed as

$$
X_{i}=\frac{1}{\sqrt{N}} \sum_{k=0}^{N-1} X_{i}(k) e^{\frac{j 2 \pi k}{N}}
$$

And the PAPR on the $i_{\text {th }}$ antenna is defined as:

$$
P A P R_{i}=10 \log _{10} \frac{\max \left\{\left|x_{i}(k)\right|^{2}\right\}}{E\left\{\left|x_{i}(k)\right|^{2}\right\}}(\mathrm{dB})
$$

The PAPR of MIMO-OFDM is defined as:

$$
P A P R=\max \left\{P A P R_{i}\right\}, \quad i \in[1,2]
$$

The complementary cumulative distribution function ( $C C D F$ ) is usually used to evaluate the PAPR performance in a MIMO-OFDM system, which physically means the probability that the PAPR value exceeds the certain threshold $P A P R_{0}$ [18]

$$
C C D F(P A P R)=P_{r}\left(P A P R>P A P R_{0}\right)
$$

\subsection{SLM scheme}

In SLM scheme, the phase distribution of the data frame is changed by multiplying random phase vectors. Fig. 1 depicts the block diagram of SLM scheme. The main idea of SLM scheme can be briefly described as follow

Assume that there are $G$ different random phase sequence vectors with length $N$

$$
P_{i}^{(\mu)}=\left[P_{i 1}^{(\mu)}, P_{i 2}^{(\mu)}, \ldots, P_{i(N-1)}^{(\mu)}\right], \quad \mu=1,2, \ldots G
$$


where $P_{i}^{(\mu)}=\exp \left(j \varphi_{i}^{(\mu)}\right), \varphi_{i}^{(\mu)}$ is evenly distributed in $\left[\begin{array}{ll}0 & 2 \pi\end{array}\right)$. However, to reduce the camputational complexity, $\varphi_{i}^{(\mu)}$ is uaually set to [0 $\left.\pi / 2 \pi 3 \pi / 2\right]$. Then, multiply the $X_{i}$ with $P_{i}^{(\mu)}$ to obtain $X_{i}^{(\mu)}$ :

$$
\begin{aligned}
X_{i}^{(\mu)} & =\left[X_{i 0}^{(\mu)}, X_{i 1}^{(\mu)}, \ldots, X_{i(N-1)}^{(\mu)}\right]=X_{i} \cdot P_{i}^{(\mu)} \\
& =\left[X_{i 0} P_{i 0}^{(\mu)}, X_{i 1} P_{i 1}^{(\mu)}, \ldots, X_{i(N-1)} P_{i(N-1)}^{(\mu)}\right]
\end{aligned}
$$

Apply IFFT to $X_{i}^{(\mu)}$ to obtain the time-domain sequences $x_{i}^{(\mu)}$

$$
x_{i}^{(\mu)}=\left[x_{i 0}^{(\mu)}, x_{i 1}^{(\mu)}, \ldots, x_{i(N-1)}^{(\mu)}\right]
$$

where

$$
x_{i 0}^{(\mu)}=\operatorname{IFFT}\left(X_{i 0}^{(\mu)}\right)
$$

Finally, the time-sequence with the smallest PAPR in $x_{i}^{(\mu)}$ is selected to be transmitted. Meanwhile, the random phase vector $P_{i}^{(\mu)}$ corresponding to $x_{i}^{(\mu)}$ is transmitted as SI. To transmit the SI in SLM, $2 N$ bits is required for each antenna.

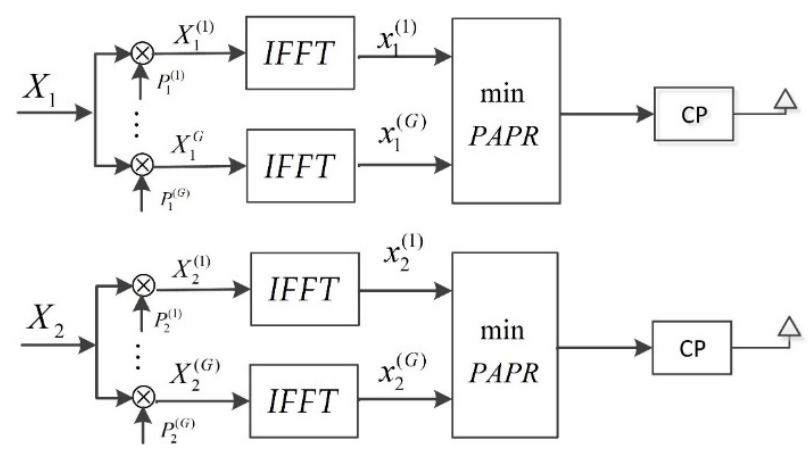

Fig. 1. Block diagram of SLM scheme

\section{AST-SLM Scheme}

In AST-SLM scheme, the ACE is done on the IFFT-transformed time-domain signal at the transmitter side at first, then various candidate sequence pairs with different PAPR are generated via weighted combination of cyclic-shifted time-domain sequences in different antennas. Compared with SLM algorithm, both the calculational complexity and SI of AST-SLM is significantly reduced. In addition, a blind detection scheme for estimating SI is proposed. And it is noteworthy that various parameters can be adjusted according to the actual system characteristics in AST-SLM scheme, which efficiently improves the flexibility and practicability.

\subsection{ACE mudule}

The clipping technology efficiently reduces the PAPR of signal, but inevitably causes signal distortion, which leads to fading in frequency band and spreading outside frequency band. However, the technique of constellation extension can largely eliminate the signal distortion caused by the clipping process [20] . Active constellation extension (ACE) can be defined as the combination of clipping technology and constellation extension process, which can be described briefly as follows: 
First , clip the time-domain sequence at the $i_{t h}$ antenna $x_{i}$, and the clipped sequence $x_{i c}$ can be written as:

$$
x_{i c}(n)= \begin{cases}A_{\max } e^{j \theta(n)} & ,\left|x_{i}(n)\right|>A_{\max } \\ x_{i}(n) & ,\left|x_{i}(n)\right| \leq A_{\max }\end{cases}
$$

where $x_{i}(n)=\left|x_{i}(n)\right| e^{j \theta(n)}$, and $A_{\max }=C R \cdot E\left\{\left|x_{i}\right|^{2}\right\}$. CR is the constant clipping rate.

Then, apply FFT to $x_{i c}$ to obtain $X_{i c}$. Checking all the $X_{i c}(k)$, as illustrated in Fig. 2, for those constellation points within the dotted line, extend them to the four standard constellation points, and for those constellation points beyond the dotted line and not in the extended area, extend them to the corresponding positions from the nearest thick solid line.

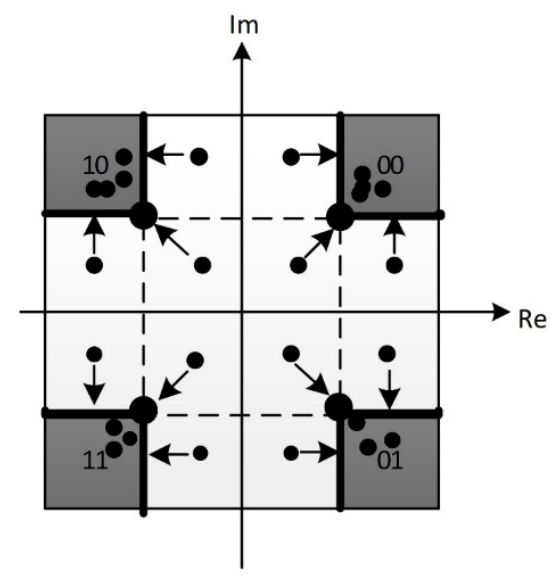

Fig. 2. Schematic diagram of QPSK constellation expansion. The extended area is illustrated in shaded area

Fig. 3 shows the constellation change of QPSK signal in ACE process. From Fig. 3(a), after clipping process, the constellation becomes divergent and the Euclidean distance between adjacent constellation points gets smaller. That is to say, the clipping process introduces equivalent noise. As shown in Fig. 3(b), in the extended constellation diagram, the Euclidean distance between adjacent constellation points is increased and the noise is effectively reduced.

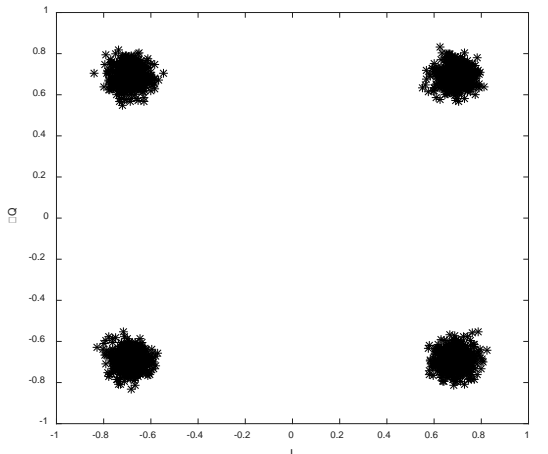

(a)

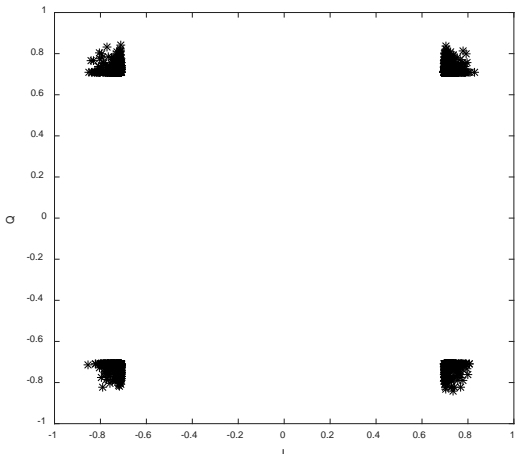

(b)

Fig. 3. Constellation change of QPSK signal in clipping-constellation-expansion 


\subsection{Joint space-time selective mapping mudule}

Again consider the two-antenna MIMO-OFDM system mentioned above. We assume that the time-domain sequences generated at antenna 1 and antenna 2 after ACE operation are $x_{1}$ and $x_{2}$, respectively. In multicarrier systems, the centralized distribution of subcarrier phases can easily lead to large power peaks [21]. The phase distribution of those new sequences obtained by weighted combination of time-domain sequences on different antennas is more random than the original sequence, and theoretically, their PAPR may be lower [16]. Based on this, a new sequence set generated by weighting $x_{1}$ and $x_{2}$ can be denoted as

$$
Z_{0}=\frac{1}{\sqrt{n}} \sum_{i=1}^{n} \pm x_{i}, n \in[1,2]
$$

where $1 / \sqrt{\mathbf{n}}$ is the power normalization weighting factor. Since the sign of the time-domain sequence does not affect the PAPR, there are actually only four sequences with different PAPR in $\boldsymbol{Z}(0)$, therefore, we correct it as follow:

$$
Z_{0}=\left\{x_{1}, x_{2}, \sqrt{1 / 2}\left(x_{1}+x_{2}\right), \sqrt{1 / 2}\left(x_{1}-x_{2}\right)\right\}
$$

Furthermore, to produce more candidate sequences, we cyclic-shift $x_{i}$ with a certain cyclic shift factor $w_{i}$ to obtain $x_{i w_{i}}$ as follow:

$$
\begin{aligned}
x_{i w_{i}} & =\operatorname{cicshift}\left(x_{i}, w_{i}\right) \\
& =\left\{x_{i}\left(w_{i}\right), x_{i}\left(w_{i}+1\right), \cdots, x_{i}(N-1), x_{i}(0), \cdots, x_{i}(w i-1)\right\}, w_{i} \in[0,1, \cdots, W-1]
\end{aligned}
$$

where cicshift(·) represents the cyclic shift operation, $W$ is the maximum shift factor. And then the set of candidate sequence can be further expanded by weighted combination of $x_{i w_{i}}$ as Eq. (13), which can be expressed as follow:

$$
Z_{w_{1} W+w_{2}}=\left\{x_{1 w_{1}}, x_{2 w_{2}}, \sqrt{1 / 2}\left(x_{1 w_{1}}+x_{2 w_{2}}\right), \sqrt{1 / 2}\left(x_{1 w_{1}}-x_{2 w_{2}}\right)\right\}
$$

Hence, $W^{2}$ candidate sequence sets can be generated.

$$
Z=\left[\begin{array}{c}
Z_{0} \\
\cdots \\
\cdots \\
Z_{w_{1} W+w_{2}}
\end{array}\right]=\left[\begin{array}{cccc}
x_{1} & x_{2} & \sqrt{1 / 2}\left(x_{1}+x_{2}\right) & \sqrt{1 / 2}\left(x_{1}-x_{2}\right) \\
\ldots & \ldots & \ldots & \ldots \\
\ldots & \ldots & \ldots & \ldots \\
x_{1 w_{1}} & x_{2 w_{2}} & \sqrt{1 / 2}\left(x_{1 w_{1}}+x_{2 w_{2}}\right) & \sqrt{1 / 2}\left(x_{1 w_{1}}-x_{2 w_{2}}\right)
\end{array}\right]
$$

And the sequence pairs choosed from $Z_{j}$ can be written as:

$$
\left[\hat{x}_{1}, \hat{x}_{2}\right]_{l}^{j}=\left[Z_{j}(a), Z_{j}(b)\right]
$$

where $a \neq b, a, b \in[1,2,3,4]$ and $1 \leq l \leq C_{4}^{2}, 0 \leq j \leq W^{2}-1$.

Finally, we consider all the $W^{2}$ candidate emission sequence sets, thus $C_{4}^{2} W^{2}$ sequence pairs with different PAPR can be obtained, and the one with the smallest PAPR is selected as the final transmission sequence pair 


$$
\begin{aligned}
{\left[\hat{x}_{1}, \hat{x}_{2}\right]_{\text {final }} } & =\arg \min _{\left[\hat{x}_{1}, \hat{x}_{2}\right]_{l}^{j}}\left\{\operatorname{PAPR}\left[\hat{x}_{1}, \hat{x}_{2}\right]_{l}^{j}\right\} \\
& =\left[\begin{array}{ll}
x_{1 \hat{w}_{1}} & \left.x_{2 \hat{w}_{2}}\right]
\end{array}\right] \\
& =\left[\begin{array}{lll}
x_{1 \hat{w}_{1}} & x_{2 \hat{w}_{2}}
\end{array}\right]\left[\begin{array}{ll}
b_{11} & b_{12} \\
b_{21} & b_{22}
\end{array}\right]
\end{aligned}
$$

where $b_{11}, b_{12}, b_{21}, b_{22} \in[0, \pm 1, \pm 1 / \sqrt{2}], \hat{w}_{1}$ and $\hat{w}_{2}$ denote the cyclic shift factor of $x_{1}$ and $x_{2}$ in $\left[\hat{x}_{1}, \hat{x}_{2}\right]_{\text {final }}$, respectively. In order to correctly restore $\left[x_{1}\left(\hat{w}_{1}\right) x_{2}\left(\hat{w}_{2}\right)\right]$ at the receiver side, $B$ is the only SI required to be transmitted. And to restore $x_{1}$ and $x_{2}$, the cyclic shift factors $\left[\hat{w}_{1} \hat{w}_{2}\right]$ are generally transmitted as SI too.

\subsection{The proposed blind detection scheme}

Under the condition that the complexity of the receiver is not a limit for system design, we propose a blind detection scheme to estimate $\left[\hat{w}_{1} \hat{w}_{2}\right]$, which bolck diagram is shown in Fig. 4 and can be described as follow.

According to the property of Fourier transform, the cyclic shift process on the time-domain sequence $x$ equates to the phase rotation in frequency-domain sequence $X$ [22], which can be denoted as:

$$
\operatorname{IFFT}\left(X(n) \cdot e^{j 2 \pi w(n-1) / N}\right)=\operatorname{cicshif}(x, w)
$$

Assume that the cyclic-shifted time-domain sequence $\left[x_{1 \hat{w}_{1}} X_{2 \hat{w}_{2}}\right]$ is restored and the number of subcarriers is large enough, the frequency-domain sequences is given as follow:

$$
\hat{X}_{i}=\operatorname{FFT}\left(x_{1 \hat{w}_{1}}\right)
$$

Then the central constellation points of $\hat{X}_{i}$ can be written as:

$$
\hat{X}_{i}^{\prime}=\left[\hat{X}_{i 0}{ }^{\prime}, \hat{X}_{i 1}{ }^{\prime}, \ldots, \hat{X}_{i M-1}{ }^{\prime}\right]
$$

where $M$ is the order of the modulation. For example, when a power-normalized QPSK signal has passed through an AWGN channel, its constellation diagram is not a standard QPSK any more, but its central constellation points can be approximately written as

$$
1 / \sqrt{2}[1+i,-1+i,-1-i, 1-i], i=\sqrt{-1}
$$

Suppose that the candidate set of cyclic shift factor is known to the receiver side, which is $[0, \ldots, W-1]$. The $W$ anti-rotation sequences can be obtained by the reverse phase rotation of $\hat{X}_{i}^{\prime}$ according to Eq. (19)

$$
\hat{X}_{i k}^{\prime}=\hat{X}^{\prime} e^{-j 2 \pi k / N}, 0 \leq k \leq W-1
$$

where $X^{\prime}$ is the standard central constellation points of the adopted modulation. There must be an anti-rotation sequence which have the smallest Euclidean distance with $X_{i}^{\prime}$. Thus, the estimation of $\left[\hat{w}_{1} \hat{w}_{2}\right]$ can be described as:

$$
\bar{w}_{i}=\arg \min _{0 \leq k \leq W-1} \sum_{d=1}^{M}\left|\hat{X}_{i k}{ }^{\prime}(d)-X^{\prime}(d)\right|^{2}
$$

The most innovative feature of this blind detection scheme is the estimation of cyclic shift factors in the receiver side. So $B$ is the only SI required to be transmitted. Although the computational complexity in receiver is increase, but the complexity of the whole system is reduced compared with SLM, which will be detailedly explained in Section 4. 


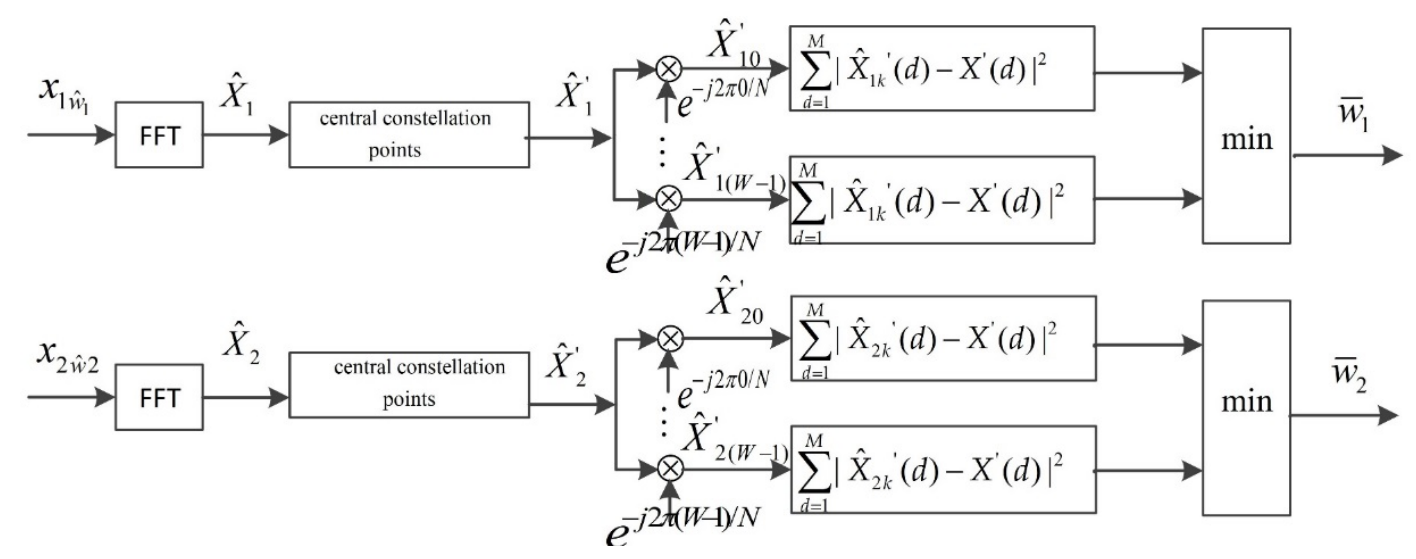

Fig. 4. Block diagram of the proposed blind detection scheme for estimating cyclic shift factors

\subsection{The transmitter side of AST-SLM}

The Block diagram of AST-SLM scheme is shown as Fig. 5, and the specific steps of the AST-SLM in transmitter side are summarized as follows

Step 1 Map the data bit stream to the certain modulation constellation (MPSK/MQAM), then perform STBC encoding to obtain the frequency-domain sequence on different antennas.

Step 2 Set the clipping rate $C R$ and the maximum number of ACE iteration $M_{t}$, and set the current iteration number $m=1$.

Step 3 Apply IFFT transformation on $X_{i}\left(\hat{X}_{i}\right)$ to obtain the time-domain sequence $X_{i}\left(\hat{x}_{i}\right)$, then implement clipping operation as Eq. (11).

Step 4 Turn the switch $K_{i}$ to the 1, perform FFT transformation on $X_{i}\left(\hat{x}_{i}\right)$ to obtain a new frequency-domain sequence $\hat{X}_{i}$, and perform constellation expansion on $\hat{X}_{i}$, set $m=m+1$.

Step 5 Repeat step 3 and step 4 till $m=M_{t}$.

Step 6 Turn the switch $K_{i}$ to the 2, and oversample $X_{i}$ with the oversample rate $L$,

$$
x_{i}=\operatorname{IFFT}\left[\hat{X}_{i O}\right]
$$

Where

$$
\hat{X}_{i O}=\left[\hat{X}_{i}(0), \ldots \hat{X}_{i}(N / 2-1) \underset{(L-1) N}{0,0, \ldots 0, \hat{X}_{i}}(N / 2+1), \ldots \hat{X}_{i}(N-1)\right]
$$

Step 7 Cyclic-shift $x_{i}$ with shift factor $w_{i}, w_{i} \in[0,1, \cdots W-1]$ to get $x_{1 w_{1}}$ and $x_{2 w_{2}}$ using Eq. (14);

Step 8 Weight the $X_{1 w_{1}}$ and $X_{2 w_{2}}$ to get a new sequence set $Z_{w_{1} W+w_{2}-1}$ using Eq. (15);

Step 9 Choose the sequence pair with the smallest PAPR in $Z_{p}, 0 \leq p \leq W^{2}-1$ as the transmission sequence pair $\left[\hat{x}_{1}, \hat{x}_{2}\right]$. 


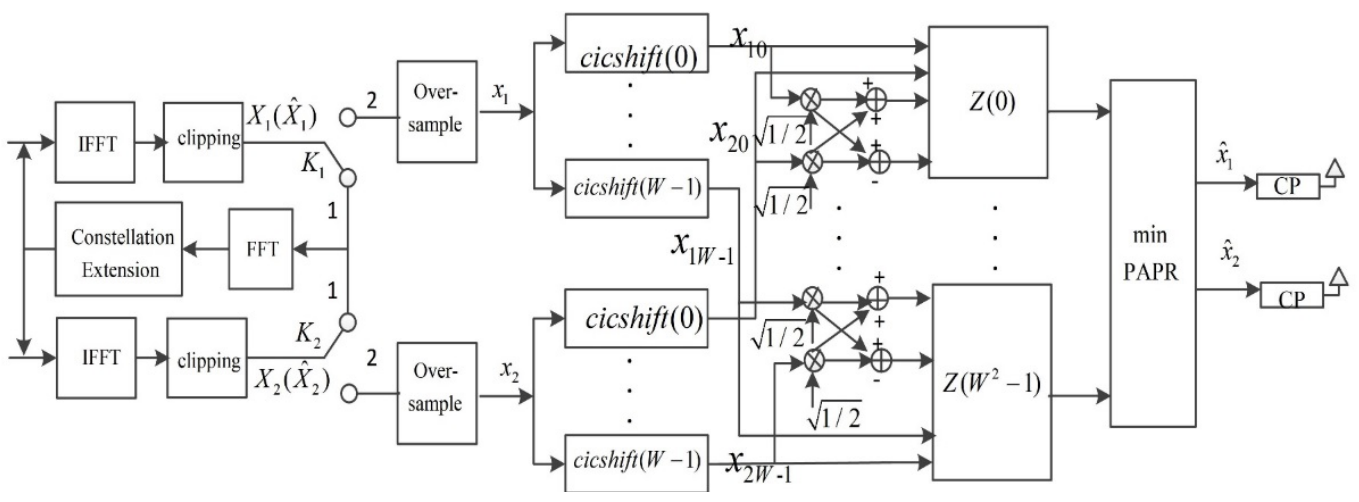

Fig. 5. Block diagram of the proposed AST-SLM scheme

\subsection{The receiver side of AST-SLM}

After channel transmission, the sequence pair in receivier side is denoted as $\left[y_{1}, y_{2}\right]$, the relationships between $\left[y_{1}, y_{2}\right]$ and the transmitted sequence pair $\left[\hat{x}_{1}, \hat{x}_{2}\right]$ can be expressed as:

$$
\left\{\begin{array}{l}
y_{1}=\hat{x}_{1} \otimes h_{11}+\hat{x}_{2} \otimes h_{21}+n_{1} \\
y_{2}=\hat{x}_{1} \otimes h_{12}+\hat{x}_{2} \otimes h_{22}+n_{2}
\end{array}\right.
$$

where $h_{i j}(i, j=1,2)$ represents the impulse response of the channel, and $n_{i}(i=1,2)$ represents the channel noise. The sequence pair obtained after channel equalization is written as $\left[\hat{x}_{1}^{*}, \hat{x}_{2}^{*}\right]$

$$
\left\{\begin{array}{l}
\hat{x}_{1}^{*}=\hat{x}_{1}+n_{1}{ }^{*} \\
\hat{x}_{2}{ }^{*}=\hat{x}_{2}+n_{2}{ }^{*}
\end{array}\right.
$$

where $n_{i}^{*}, i \in[1,2]$ is the sum of channel noise and channel equalization equivalent error noise. And the recovered cyclic-shifted sequence is given as:

$$
\left[x_{1 w_{1}}^{\prime}, x_{2 w_{2}}^{\prime}\right]=\left[\hat{x}_{1}^{*}, \hat{x}_{1}^{*}\right] B^{-1}
$$

Finally, the original sequence can be restored by inversely cyclic-shifting the $\left[x_{1 w_{1}}^{\prime}, x_{2 w_{2}}^{\prime}\right]$ with the estimated $\left[\bar{w}_{1}, \bar{w}_{2}\right]$, that is

$$
\bar{x}_{i}=\left[x_{i w_{1}}^{\prime}\left(\bar{w}_{1}\right), x_{i w_{1}}^{\prime}\left(\bar{w}_{1}+1\right), \ldots x_{i w_{1}}^{\prime}(L N-1), x_{i w_{1}}^{\prime}(0), \ldots x_{i w_{1}}^{\prime}\left(\bar{w}_{1}-1\right)\right]
$$

Now we consider the SI needed in AST-SLM. More specifically, to transmit $B$ and $\left[\hat{w}_{1} \hat{w}_{2}\right]$, only 8 and $2 \log _{2} W$ bits is needed, respectively. If the $\left[\hat{w}_{1} \hat{w}_{2}\right]$ is estimated by the blind detection scheme, then $B$ is the only SI of AST-SLM, that is to say, only 8 bits is required. Here we define SI reduction ratio (SRR), which can be expressed as:

$$
S R R=\left(1-\frac{S I \text { of } A S T-S L M}{\text { SI of } S L M}\right) \times 100 \% \geq\left(1-\frac{8+2 \log _{2} W}{4 N}\right) \times 100 \%
$$

Since $N \geq 64$ and $W \leq N$ in practical MIMO-OFDM systems, thus $S R R \geq 92.1 \%$ can be guaranteed. In other words, AST-SLM further reduces the SI by at least $92.1 \%$ compared to SLM , so the higher spectrum utilization can be realized in the system. 


\section{Complexity Analysis}

In this section, we analyse the computational complexity of AST-SLM, SLM, ACE and PTS. It is noteworthy that the computational complexity of AST-SLM depends on whether the cyclic shift factors $\left[w_{1}, w_{2}\right]$ are known to the receiver, so we discuss the two situations in AST-SLM: cyclic shift factors are known (AST-SLM1) and unknown (AST-SLM2). To simplify the model, we only consider the complex multiplication (CM) and complex addition (CA) as indication of computational complexity.

\subsection{Calculational complexity of transmitter}

Assum that the number of subcarriers is $N$ and the oversample rate is $L$, the computational complexity for a $N$-points IFFT/FFT transformation at the transmitter are $N \log _{2} N / 2$ CMs and $N \log _{2} N$ CAs. We firstly consider the ACE module of the AST-SLM scheme. In the first $M_{t}-1$ iterations, only one $N$-point IFFT and FFT are performed each iteration. Whereas, only one $L N$-points IFFT is needed in the last iteration. Therefore, $\left(M_{t}-1\right) N \log _{2} N+L N \log _{2} L N$ CMs and $2\left(M_{t}-1\right) N \log _{2} N+2 L N \log _{2} L N$ CAs are required in the ACE module. Next, we consider the joint space-time selective mapping module. $4 W^{2} L N$ CAs are required to weighted-combine the cyclic-shifted sequences on different antennas. Thus, the number of CM and CA required in tranmitter of AST-SLM scheme are $\left(M_{t}-1\right) N \log _{2} N+L N \log _{2} L N$ and $2\left(M_{t}-1\right) N \log _{2} N+2 L N\left(\log _{2} L N+2 W^{2}\right)$, respectively.

Under the same conditions that the number of alternative sequences is $W^{2}$, the number of CM and CA required in SLM are $W^{2} L N \log _{2} L N$ and $2 W{ }^{2} L N \log _{2} L N$, respectively. And for ACE , they are $\left(M_{t}-1\right) N \log _{2} N+L N \log _{2} L N$ and $2\left(M_{t}-1\right) N \log _{2} N+2 L N \log _{2} L N$. However, for PTS, $W^{2}=V^{P}, V$ is the partition number of OFDM signal, and $P=4$ is the elements number of phase set $\varphi_{i}^{(\mu)}=[0 \pi / 2 \pi 3 \pi / 2]$. So the number of CM and CA required in PTS are $\sqrt{W} L N \log _{2} L N$ and $2 \sqrt{W} L N \log _{2} L N$, respectively.

\subsection{Calculational complexity of receiver}

Under the condition that the SI $B$ is known, in the receiver side of AST-SLM, the received sequence pair $\left[\hat{x}_{1}^{*}, \hat{x}_{1}^{*}\right]$ is firstly operated based on Eq. (29), then the cyclic-shifted sequences $\left[x_{1 w_{1}}^{\prime}, x_{2 w_{2}}^{\prime}\right]$ are recovered, and the computational complexity for this step is $2 L N$ CAs.

When cyclic shift factors $\left[w_{1}, w_{2}\right]$ is known to the receiver as SI, to restore $x_{i}$, reverse cyclic shift operation is applied to $\left[x_{1 w_{1}}^{\prime}, x_{2 w_{2}}^{\prime}\right]$ to obtain $\bar{x}_{i}$ as Eq. (30). This step do not require any CA or CM. Then, FFT is performed on $\bar{x}_{i}$ to obtain the restored frequency-domain sequences $\bar{X}_{i}$, where $L N \log _{2} L N$ CMs and $2 L N \log _{2} L N$ CAs are required. Thus, $L N \log _{2} L N$ CMs and $2 L N\left(1+\log _{2} L N\right)$ CAs are required in AST-SLM.

If $\left[w_{1}, w_{2}\right]$ is unknown to the receiver, FFT is first performed on $\left[x_{1 w_{1}}^{\prime}, x_{2 w_{2}}^{\prime}\right]$ to obtain the frequency-domain sequences with phase deflection $\left[X_{1 w_{1}}^{\prime}, X_{2 w_{2}}^{\prime}\right]$, where $L N \log _{2} L N$ CMs and 
$2 L N \log _{2} L N$ CAs are required. To estimate $\left[w_{1}, W_{2}\right], 2 W N$ CMs are needed for the proposed blind detection scheme. Thus, $N\left(2 W+L \log _{2} L N\right)$ CMs and $2 L N\left(1+\log _{2} L N\right)$ CAs are required.

When SI is known, the other three algorithms have the same computational complexity at the receiver, $L N \log _{2} L N$ CMs and $2 L N \log _{2} L N$ CAs.

Table 1 summarizes the computational complexity of the above four schemes. As can be seen, many parameters affect the computational complexity. And the value of subcarrier number $N$ and maximun cyclic shift factor $W$ are closely related to the computational complexity. Their relationships are shown in Fig. 6 and Fig. 7.

As illustrated in Fig. 6, as $N$ increases, the computational complexity of the four schemes increase both in the transmitter side and the receiver side. Compared to SLM and PTS, the computational complexity in the transmitter side of AST-SLM and ACE is decreased greatly. In the receiver side, computational complexity of AST-SLM is slightly increased when cyclic shift factor is not transmitted as SI. This is because the estimation of cyclic shift factor requires computational effort.

Table 1. Computational complexity of the transmitter side and receiver side

\begin{tabular}{|c|c|c|c|c|}
\hline \multirow{2}{*}{ Algorithm } & \multicolumn{2}{|c|}{ Transmitter } & \multicolumn{2}{|c|}{ Receiver } \\
\hline & CM & $\mathrm{CA}$ & $\mathrm{CM}$ & $\mathrm{CA}$ \\
\hline $\begin{array}{c}\text { AST-SLM1 } \\
\left(\left[w_{1}, w_{2}\right] \text { is known }\right)\end{array}$ & \multirow{2}{*}{$\begin{array}{l}\left(M_{t}-1\right) N \log _{2} N \\
+L N \log _{2} L N\end{array}$} & \multirow{2}{*}{$\begin{array}{l}2\left(M_{t}-1\right) N \log _{2} N \\
+2 L N\left(\log _{2} L N+2 W^{2}\right)\end{array}$} & $L N \log _{2} L N$ & \multirow{2}{*}{$2 L N\left(1+\log _{2} L N\right)$} \\
\hline $\begin{array}{c}\text { AST-SLM2 } \\
\left(\left[w_{1}, w_{2}\right] \text { is unknown }\right)\end{array}$ & & & $N\left(2 W+L \log _{2} L N\right)$ & \\
\hline SLM & $W^{2} L N \log _{2} L N$ & $2 W^{2} L N \log _{2} L N$ & $L N \log _{2} L N$ & $2 L N \log _{2} L N$ \\
\hline ACE & $\begin{array}{l}\left(M_{t}-1\right) N \log _{2} N \\
+L N \log _{2} L N\end{array}$ & $\begin{array}{l}2\left(M_{t}-1\right) N \log _{2} N \\
+2 L N \log _{2} L N\end{array}$ & $L N \log _{2} L N$ & $2 L N \log _{2} L N$ \\
\hline PTS & $\sqrt{W} L N \log _{2} L N$ & $2 \sqrt{W} L N \log _{2} L N$ & $L N \log _{2} L N$ & $2 L N \log _{2} L N$ \\
\hline
\end{tabular}
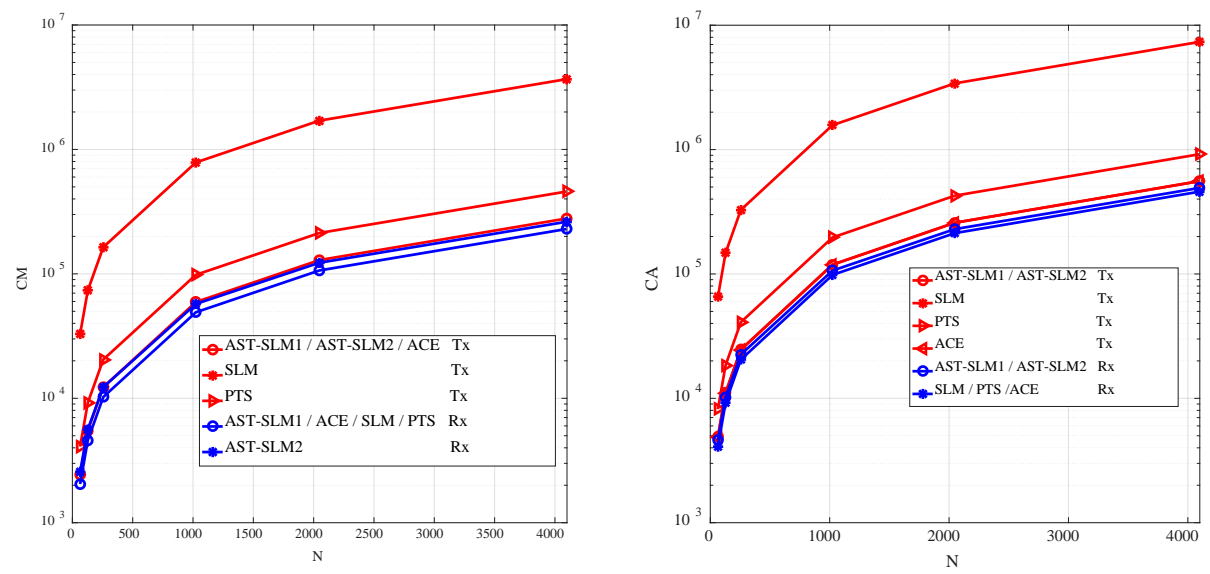

Fig. 6. Relationships between the complexity and $N$ when $W=4, L=4, M t=2$ 
Fig. 7 shows that, with the increase of $W$, the computational complexity of the four schemes increase in the transmitter side, but almost stay unchanged in the receiver side(when cyclic shift factor is transmitted as SI). Under the situation that cyclic shift factor is not transmitted as SI, AST-SLM need more computational complexity in the receiver side than other schemes. However, in the transmitter side, the computational complexity (especially CM) of AST-SLM is greatly reduced compared to SLM and PTS.
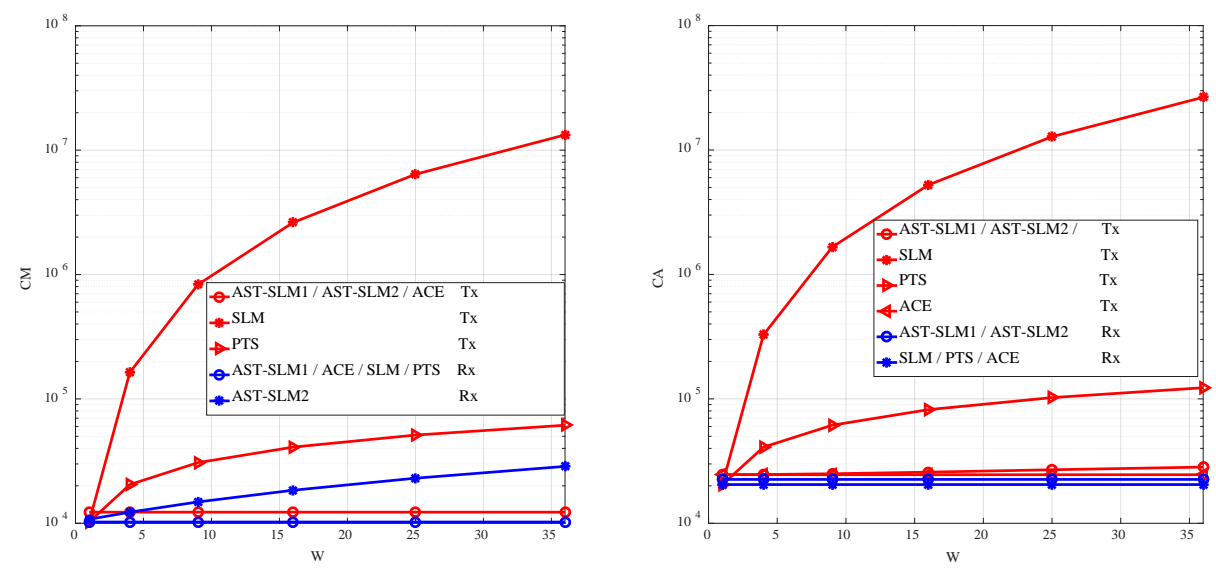

Fig. 7. Relationships between the complexity and $W$ when $N=256, L=4, M t=2$

We defined computational complexity reduction ratio(CCRR) to get better comparison of computational complexity between AST-SLM and SLM. The CCRR can be writed as:

$$
C C R R=\left(1-\frac{\text { complexity of AST }-S L M}{\text { complexity of } S L M}\right) \times 100 \%
$$

where the complexity includes the complexity of transmitter and receiver.

Table 2 gives the CCRR under different values of $N$ and $W$, and we set $L=4, M t=2$. As can be seen, all the CCRR values are greater than $86 \%$, some CCRR values are even close to $100 \%$. This proves once again that the computational complexity of AST-SLM is much lower than SLM.

Table 1. CCRR of AST-SLM

\begin{tabular}{|c|c|c|c|c|c|c|c|}
\hline \multicolumn{2}{|c|}{ State of $\left[w_{1}, w_{2}\right]$} & \multicolumn{2}{c|}{$N=64$} & \multicolumn{2}{c|}{$N=256$} & \multicolumn{2}{c|}{$N=1024$} \\
\cline { 3 - 8 } & & CM & CA & CM & CA & CM & CA \\
\hline \multirow{3}{*}{ known } & $W=4$ & $87.9 \%$ & $86.2 \%$ & $87.5 \%$ & $86.4 \%$ & $87.2 \%$ & $86.5 \%$ \\
\cline { 2 - 8 } & $W=10$ & $98.1 \%$ & $97.6 \%$ & $98.0 \%$ & $97.7 \%$ & $97.9 \%$ & $97.7 \%$ \\
\cline { 2 - 8 } & $W=16$ & $99.2 \%$ & $99.0 \%$ & $99.2 \%$ & $99.1 \%$ & $99.2 \%$ & $99.1 \%$ \\
\hline \multirow{3}{*}{ unknown } & $W=4$ & $86.3 \%$ & $86.2 \%$ & $86.2 \%$ & $86.4 \%$ & $86.2 \%$ & $86.5 \%$ \\
\cline { 2 - 8 } & $W=10$ & $97.4 \%$ & $97.6 \%$ & $97.5 \%$ & $97.7 \%$ & $97.5 \%$ & $97.7 \%$ \\
\cline { 2 - 8 } & $W=16$ & $98.8 \%$ & $99.0 \%$ & $98.9 \%$ & $99.1 \%$ & $98.9 \%$ & $99.1 \%$ \\
\hline
\end{tabular}




\section{Simulation Results and Analysis}

The PAPR and BER performance of the above mentioned schemes is simulated in this section. The parameters considerd in the MATLAB simulation are given as Table 2.

\subsection{PAPR performance}

Fig. 8 is the CCDF curve of AST-SLM scheme under different parameter settings. As shown, the PAPR performance of AST-SLM is closely related to the maximum shift factor $w$, the clipping rate $C R$, and the number of ACE iterations $M_{t}$. Specifically, the PAPR performance is positively correlated with the values of $W$ and $M_{t}$, and negatively correlated with the values of $C R$. It is because that the PAPR reduction performance is improved with $M_{t}$ increasing and $C R$ decreasing, and the number of alternative transmision sequence pairs is increased as $W$ increase. For example, when $W=4$ and $C R=3$, the $10^{-3}$ PAPR is about $0.1 \mathrm{~dB}$ reduced when increasing $M_{t}=2$ to 4 . When $W=4$ and $M_{t}=2$, the $10^{-3} \mathrm{PAPR}$ is approximately $0.35 \mathrm{~dB}$ reduced when decreasing $C R=4$ to 3 . And when $M_{t}=2$ and $C R=3$, the $10^{-3}$ PAPR is $0.38 \mathrm{~dB}$ reduced when increasing $W=4$ to 10 . However, $M_{t}$ and $C R$ can not be too large or too small, otherwise, the signal distortion is serious. In addition, the larger the $w$ and $M_{t}$ are, the more the computational complexity is increased, which lead to the reducing of the real-time performance of the scheme. Therefore, in practical applications, the algorithm parameters should be flexibly configured according to the real-time requirements of systems.

Table 3. Parameters in simulation

\begin{tabular}{|c|c|}
\hline Parameter & Specification \\
\hline System & $\begin{array}{c}\text { STBC MIMO-OFDM system with two transmit } \\
\text { antennas }\end{array}$ \\
\hline Channel & fading multipath channel with Doppler frequency shift \\
\hline Subcarriers number $(N)$ & 1024 \\
\hline Oversampling rate $(L)$ & 4 \\
\hline Clipping rate $(C R)$ & 2,3 \\
\hline Maximum shift factor $(W)$ & $4,10,16$ \\
\hline partition number of PTS $(V)$ & 2,4 \\
\hline Number of ACE iterations $\left(M_{t}\right)$ & 2,4 \\
\hline Random phase in SLM/PTS $\left(\varphi_{i}^{(\mu)}\right)$ & $0, \pi / 2, \pi, 3 \pi / 2$ \\
\hline Modulation & QPSK \\
\hline Time of simulations & 10000 \\
\hline
\end{tabular}

Fig. 9 indicates the CCDF curves of the four schemes. From the results, the CCDF curve of AST-SLM is steeper than SLM, ACE and PTS, indicating that the PAPR reduction performance of AST-SLM is efficiently improved. Let $W$ be the same value, when $C C D F=10^{-3}$, the PAPR of AST-SLM is $0.5 \mathrm{~dB}$ lower than SLM and PTS. Moreover, AST-SLM also outperforms ACE in PAPR performance. The reason is that, in AST-SLM, the PAPR is drastically reduced twice. The first time is achieved by ACE process and the second 
time is realized by joint space-time selective mapping.

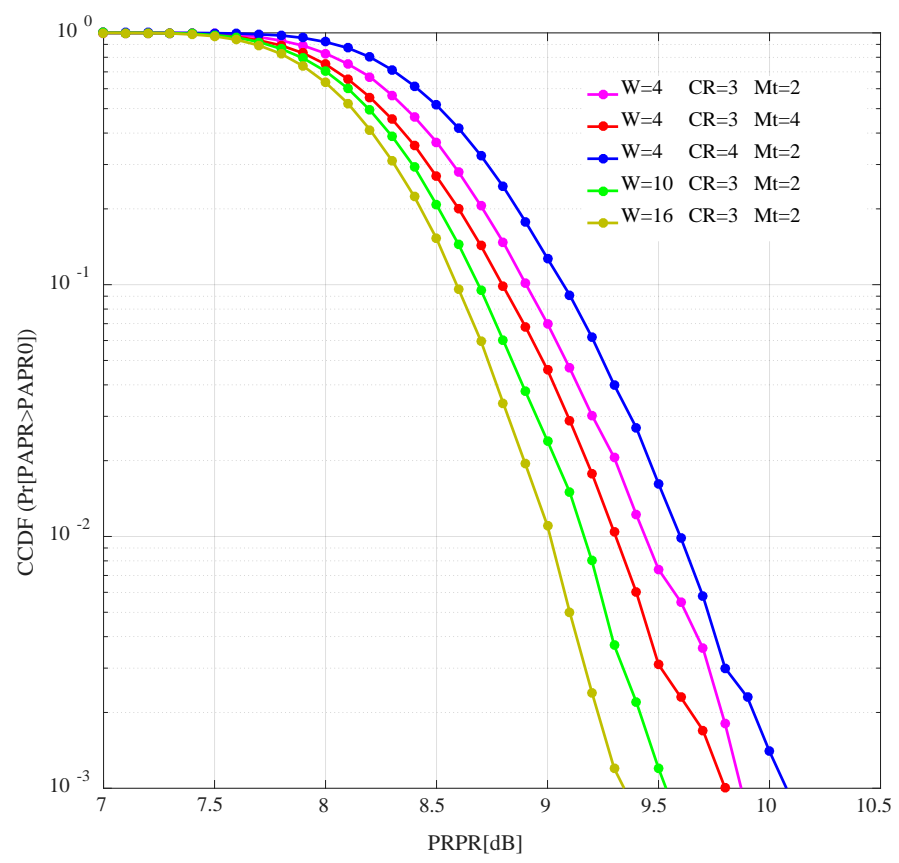

Fig. 8. CCDF curve of AST-SLM with different parameters

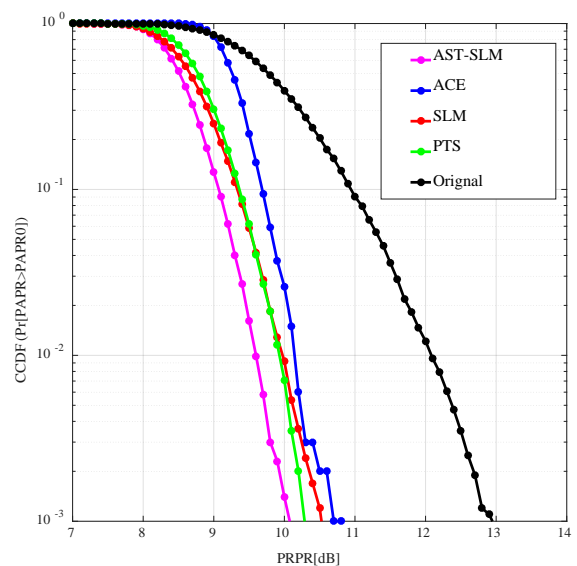

(a) $W=4$

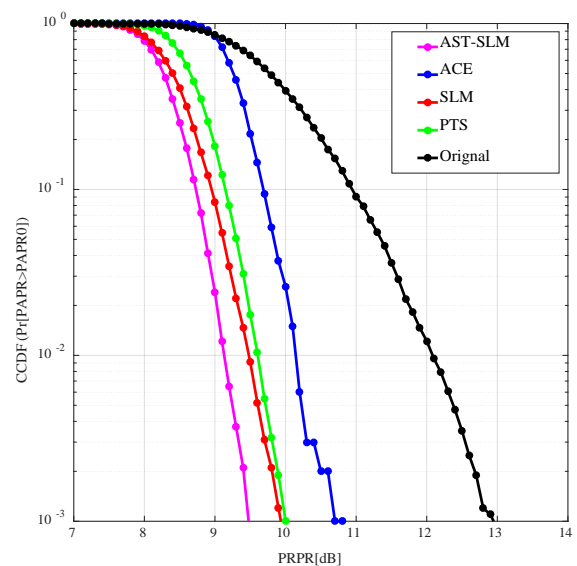

(b) $W=16$

Fig. 9. CCDF curve of different methods $M t=2, C R=4$ for AST-SLM)

\subsection{BER performance}

Fig. 10 shows the BER performance curves of the four schemes in the receiver side when passing through the fading multipath channel with Doppler frequency shift. The time delay of the channel is [0 0.0120 .0240 .0360 .0480 .06 ] $T$, where $T$ is the symbol period, and the path energy is [1.0 0.6095 0.4945 0.394 0.2371 0.19], and the maximum Doppler shift is set to $0.01 \Delta f$, where $\Delta f$ is the subcarrier frequency spacing. It is noteworthy that the BER performance of the AST-SLM is almost unaffected by the maximum shift factor $W$, but is 
slightly worse than the SLM and PTS. And the reason is twofold: on the one hand, the ACE module introduces the signal distortion to the system, on the other hand, the signal variance increases in the process of generating the candidate sequence pairs by weighted combination between sequence on different antennas, which introduces more noise than SLM and PTS. However, there are some remedial measures can be done to reduce the signal distortion which introduced by ACE, such as filtering. This will be the focus of our next research.

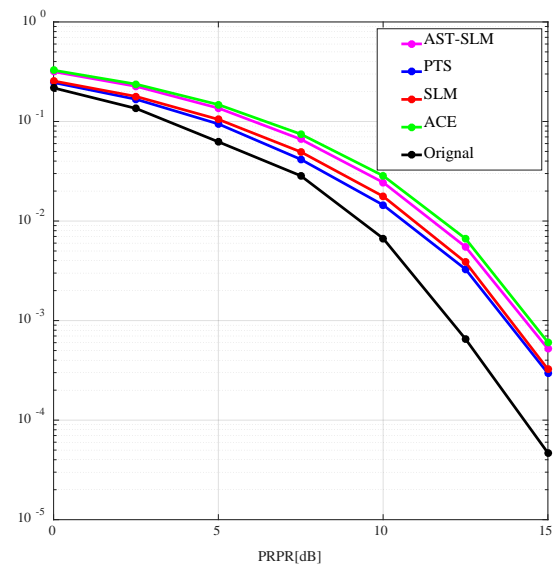

(a) $W=4$

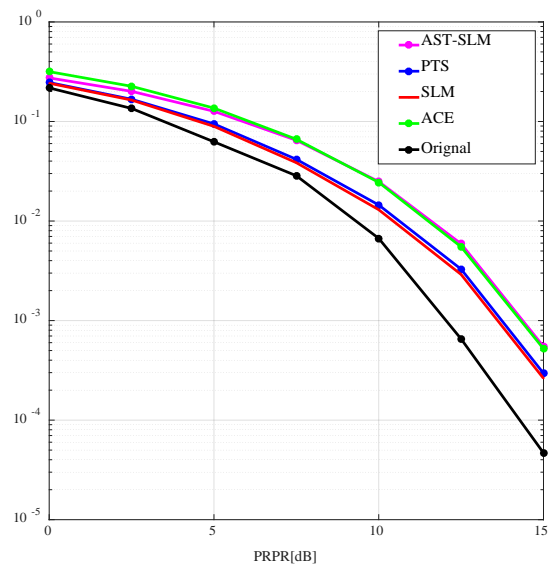

(b) $W=16$

Fig. 10. BER curve of different schemes $(M t=2, C R=4$ for AST-SLM)

\section{Conclusion}

In this paper, a low-complexity method based on active constellation extension and joint space-time SLM (AST-SLM) for reducing PAPR in Alamouti STBC MIMO-OFDM systems is proposed. The proposed method can obtain better PAPR reduction performance and require less SI than SLM, PTS and ACE. And compared to SLM and PTS, the computational complexity of AST-SLM is greatly reduced in transmitter side. In addition, a blind detection scheme to estimate SI is proposed, which greatly reduced the SI. Though the BER performance of AST-SLM is slightly worse than SLM and PTS, but AST-SLM still outperforms them by its greater PAPR reduction performance and lower computational complexity.

\section{References}

[1] Yu-Kuan Chang, Fang-Biau Ueng, Yi-Wei Jhang, "Turbo MIMO-OFDM Receiver in Time-Varying Channels,” KSII Transactions on Internet \& Information Systems, Vol. 12, No. 8, pp. 3704-3724, August 2018. Article (CrossRef Link).

[2] "Ieee approved draft standard for it - telecommunications and information exchange between systems - LAN/MAN - specific requirements - part 11: Wireless LAN medium access control and physical layer specifications- amd 4: Enhancements for very high throughput for operation in bands below 6ghz," IEEE P802.11ac/D7.0, pp. 1-456, December, 2013. Article (CrossRef Link).

[3] "IEEE standard for local and metropolitan area networks part 16: Air interface for fixed and mobile broadband wireless access systems amendment 2: Physical and medium access control layers for combined fixed and mobile operation in licensed bands and corrigendum 1," IEEE Std 802.16e-2005 and IEEE Std 802.16-2004/Cor 1-2005 (Amendment and Corrigendum to IEEE Std 802.16-2004), pp. 1-822, 2006. Article (CrossRef Link). 
[4] Shu-Ming Tseng, Yung-Fang Chen, “Average PSNR Optimized Cross Layer User Grouping and Resource Allocation for Uplink MU-MIMO OFDMA Video Communications,” IEEE Access, vol. 6, pp. 50559-50571, 2018. Article (CrossRef Link).

[5] Sampath H, Talwar S, Tellado J, et al., "A fourth-generation MIMO-OFDM broadband wireless system: design, performance, and field trial results,” IEEE Communications Magazine, vol. 40, no. 9,pp. 143-149, 2002. Article (CrossRef Link).

[6] Hao Y H, "Research on MIMO-OFDM Technology Based on PDM Method for 5G Systems," Mobile Communications, 2016. Article (CrossRef Link).

[7] S. M. Alamouti, “A simple transmit diversity technique for wireless communications,” IEEE J. Sel. Areas Commun., vol. 16, pp.1451-1458, Oct. 1998. Article (CrossRef Link).

[8] Li X, Cimini L J J, “Effects Of Clipping And Filtering On The Performance Of Ofdm,” IEEE Communications Letters, vol. 2, no. 5, pp. 131-133, 1998. Article (CrossRef Link).

[9] Bauml. R., W. Fischer. et al, "Reducing the peak-to-average power ratio of multicarrier modulation by selected mapping,” Electronics Letters, vol. 32, no.22, pp.2056-2057, 1996.

Article (CrossRef Link).

[10] Yang Z, Fang H, Pan C, "ACE with frame interleaving scheme to reduce peak-to-average power ratio in OFDM systems," IEEE Transactions on Broadcasting, vol.51, no.4, pp.571-575, 2005. Article (CrossRef Link).

[11] Kang S G, Kim J G, Joo E K, “A novel subblock partition scheme for partial transmit sequence OFDM,” IEEE Transactions on Broadcasting, vol. 45, no.3, pp.333-338, 1999. Article (CrossRef Link).

[12] More. A. P, Somani. S. B, "The reduction of PAPR in OFDM systems using clipping and SLM method," in Proc. of International Conference on Information Communication and Embedded Systems. IEEE, pp.593-597, 2013. Article (CrossRef Link).

[13] Kou. Y. J, Lu. W. S, Antoniou. A, “A New Peak-to-Average Power-Ratio Reduction Algorithm for OFDM Systems via Constellation Extension,” IEEE Trans Wireless Commun, vol.6, no.5, pp.1823-1832, 2007. Article (CrossRef Link).

[14] Jiang T, Ni C, Guan L, "A Novel Phase Offset SLM Scheme for PAPR Reduction in Alamouti MIMO-OFDM Systems Without Side Information,” IEEE Signal Processing Letters, vol.20, no.4, pp. 383-386, 2013. Article (CrossRef Link).

[15] Ayad. A. Abdulkaf, Mohamad. Yusoff. Alias, et al, “A Hybrid PAPR Reduction Scheme for Optical Wireless OFDM Communication Systems,” KSII Transactions on Internet \& Information Systems, Vol. 12, No. 3, pp. 1136-1151, March 2018. Article (CrossRef Link).

[16] Hu Wujun, Yang Lin, “An improved SLM algorithm based on cyclic shift and signal combination in MIMO-OFDM systems,” JOURNAL OF COMPUTATION, vol.36, no.4, pp. 170-177, 2015.

[17] Vijayalakshmi. M, Rama. L. R. K., "Efficient PAPR Reduction Approach for Selective Mapping in a STBC MIMO-OFDM System," International Journal of Computer Networks \& Communications, vol.10, no.3, pp.45-53, 2018. Article (CrossRef Link).

[18] Liu Fang, Wang Yong, "Peak-to-average ratio suppression algorithm for reserved subcarrier OFDM signal with joint constellation extension,” Transactions of Communications, vol.37, no.3, pp. 175-181, 2016.

[19] Yang Z , Fang H, Pan C, “ACE with frame interleaving scheme to reduce peak-to-average power ratio in OFDM systems,” IEEE Transactions on Broadcasting, 2005, vol.51, no.4, pp. 571-575, 2005. Article (CrossRef Link).

[20] Bae. K, Andrews. J. G, Powers E. J, “Adaptive active constellation extension algorithm for peak-to-average ratio reduction in OFDM,” IEEE Communications Letters, vol.14,no.1, pp.39-41, 2010. Article (CrossRef Link).

[21] Tan. M, Latinović. Z, Bar-Ness. Y, "STBC MIMO-OFDM peak-to-average power ratio reduction by cross-antenna rotation and inversion,” IEEE Communications Letters, vol.9, no.7, pp.592-594, 2005. Article (CrossRef Link).

[22] Mallat S, Hwang W L, "Singularity detection and processing with wavelets," IEEE Press, vol.38, no.2, pp.617-643, 1992. Article (CrossRef Link). 


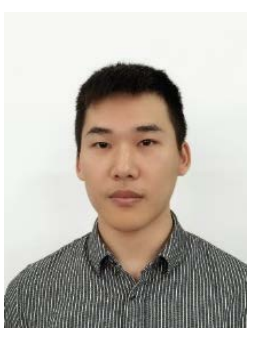

Guang Li received the B.S. degree in National Digital System Engineering and Technological Research Center, Zhengzhou, China in 2017. He is currently working towards the M.S. degree on signal processing in National Digital System Engineering and Technological Research Center. His research interests are in OFDM signal demodulation and channel estimation.

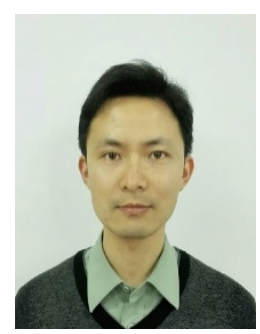

Tianyun $\mathbf{L i}$ is an associate professor of National Digital System Engineering and Technological Research Center, Zhengzhou, China. He received his PhD in communication engineering in National Digital System Engineering and Technological Research Center in 2011. His research interests includes signal processing and parameter estimation. 